\title{
Punch-Sketching E-textiles
}

\author{
Exploring Punch Needle as a Technique for Sustainable, Accessible, and Iterative Physical Prototyping \\ with E-textiles
}

\author{
Lee Jones \\ Carleton University \\ Ottawa, Ontario, Canada \\ Lee.Jones@Carleton.ca \\ Sara Nabil \\ Queen's University \\ Kingston, Ontario, Canada \\ sara.nabil@queensu.ca
}

\author{
Miriam Sturdee \\ Lancaster University \\ Lancaster, United Kingdom \\ m.sturdee@lancaster.ac.uk \\ Audrey Girouard \\ Carleton University \\ Ottawa, Ontario, Canada \\ Audrey.Girouard@Carleton.ca
}

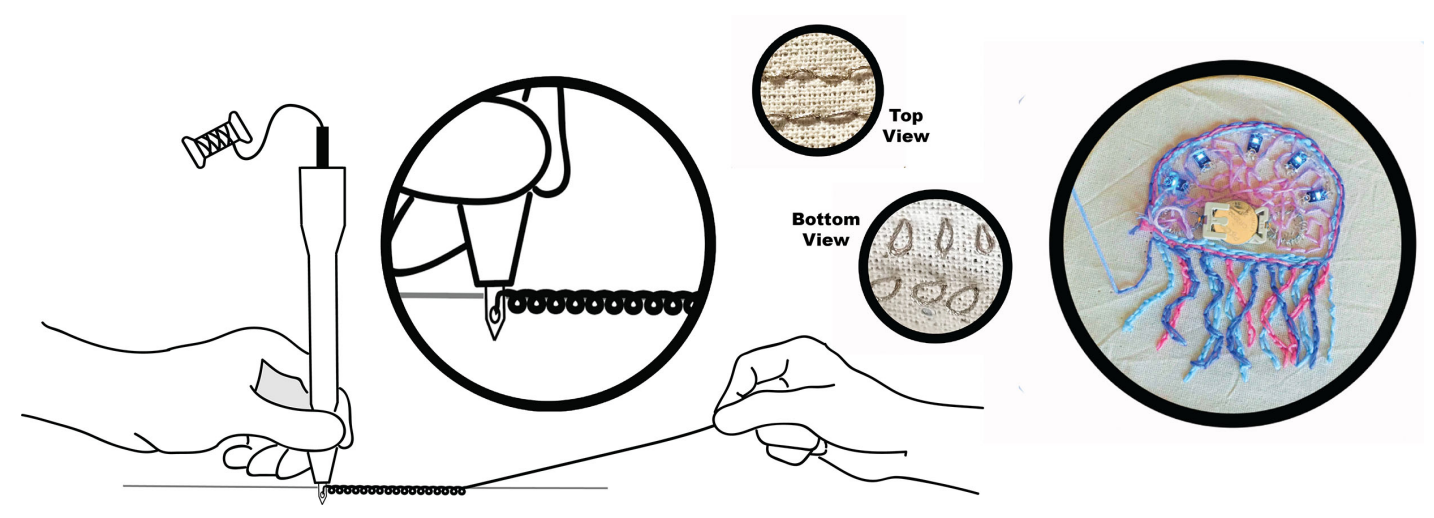

Figure 1: The Punch-Sketching e-textiles technique enables individuals to "draw" with a single conductive thread (see left hand) and then easily pull threads out to redo circuits (see right hand). The technique creates stitches on the top and loops seen from the bottom (middle). Punch-Sketching enables individuals to prototype while being creatively expressive (right).

\begin{abstract}
Tangible toolkits enable individuals to explore concepts through combining components together and taking them apart. The strength and limitation of many e-textile toolkits is that threads hold them in place, and once put together they need destructive methods to take them apart. In this paper, we propose Punch-Sketching e-textiles, a drawing technique that uses a punch needle to iteratively prototype soft circuits. The benefits of this approach is sustainability and reusability where users can easily pull out circuits without damaging the materials or creating waste, while also testing out concepts using the actual threads that will be used in the final prototype. To validate our technique, we ran three studies comparing sewing and punching e-textiles through: 1) Understanding the process with two
\end{abstract}

Permission to make digital or hard copies of all or part of this work for personal or classroom use is granted without fee provided that copies are not made or distributed for profit or commercial advantage and that copies bear this notice and the full citation on the first page. Copyrights for components of this work owned by others than the author(s) must be honored. Abstracting with credit is permitted. To copy otherwise, or republish, to post on servers or to redistribute to lists, requires prior specific permission and/or a fee. Request permissions from permissions@acm.org.

TEI '21, February 14-17, 2021, Salzburg, Austria

(C) 2021 Copyright held by the owner/author(s). Publication rights licensed to ACM. ACM ISBN 978-1-4503-8213-7/21/02 ..\$15.00

https://doi.org/10.1145/3430524.3440640 fiber artists; 2) Exploring the potential with four beginner users; and 3) Utilizing our methods further with 10 occupational therapists. Insights from these three studies include when and how to use each method, toolkit recommendations, considerations for iterative physical prototyping, sustainability, and accessibility.

\section{CCS CONCEPTS}

- Human-centered computing $\rightarrow$ User interface toolkits.

\section{KEYWORDS}

e-textiles, sketching, prototyping, toolkits, constructive assemblies, accessibility, sustainability, textiles, occupational therapy

\section{ACM Reference Format:}

Lee Jones, Miriam Sturdee, Sara Nabil, and Audrey Girouard. 2021. PunchSketching E-textiles: Exploring Punch Needle as a Technique for Sustainable, Accessible, and Iterative Physical Prototyping with E-textiles. In Fifteenth International Conference on Tangible, Embedded, and Embodied Interaction (TEI '21), February 14-17, 2021, Salzburg, Austria. ACM, New York, NY, USA, 12 pages. https://doi.org/10.1145/3430524.3440640 


\section{INTRODUCTION}

Makerspace prototyping tools, though useful for iteratively exploring and tinkering, are also known for producing large amounts of waste [15]. This is compounded in the field of e-textiles, which produces waste through both short textile lifecycles (such as fast fashion) and consumable electronic hardware components [66]. In response, researchers in Human Computer Interaction (HCI) are increasingly exploring and emphasizing the importance of sustainable use of materials and how this waste can be repurposed [15, 37, 66]. Sustainable interaction design explores how we can design with sustainability in mind from the beginning by considering what we will do with an object when it is no longer needed $[3,54]$.

One way to do this is to design objects so that their materials can be easily reused to make new things [3], as is the case with constructive assemblies. Constructive assemblies are tangible user interfaces made of modular components that can be put together, taken apart, and reassembled [26,36]. This ability to try out concepts and reuse components in a hands-on way makes constructive assemblies particularly useful for education and rapid prototyping [36] . Most constructive assemblies are inspired by tangible blocks and connect through reusable components [2]. In contrast, e-textile constructive assemblies, called e-textile toolkits in the research [50], have a tension between the ability to reuse components and the ability for creative expression [36]. More creative and expressive toolkits such as the LilyPad [10] use sewn conductive threads and produce waste through the use of consumable materials, whereas block-inspired toolkits with reusable components such as Wearable Bits [30] allow individuals to try out concepts and take them apart, but are less expressive.

In this paper, we aim to address this tension between expressivity and reuse in e-textile toolkits by proposing Punch-Sketching e-textiles, a punch-needle toolkit that enables individuals to reuse e-textile threads (Figure 1). The motivation for this research project is based on the first-author's experience as an e-textile educator running workshops with beginners, including instructing over 100 participants last year. There are two issues that this work aims to address based on the author's first-hand experience. First, unlike the Arduino beginner workshops that have reusable components such as breadboards and wires, e-textile workshops have a large amount of materials that become consumables because they are sewn in place. Reuse would be especially useful for initial experiential learning activities such as learning how to build series and parallel circuits. Second, mistakes are an important part of learning, such as the first time someone sews in an LED backwards, and the permanent nature of sewing items in place often leads participants to feel frustrated when they have to cut out their work and start again.

To solve this, we utilized the crafting method of 'punch-needle' (a crafting technique for threading into fabric membranes without sewing) for sketching and stitching re-usable e-textile circuits (Figure 2). Using this method, we ran 3 studies: 1) Interviews with fiber artists to understand the technique and tools, 2) Comparative studies with beginners, and 3) Interviews with occupational therapists to discuss tool use. This paper contributes a multidisciplinary evaluation of this adapted punch needle method and its use in accessible e-textiles crafting.
Our two main research questions for this work are:

R1: For beginners, how would Punch-Sketching affect the process and experience of prototyping e-textiles compared to sewing etextiles with a sewing needle?

R2: What are the impacts and benefits of the Punch-Sketching in terms of reuse of materials, sustainability, accessibility, and inclusive design?

\section{RELATED WORK}

A sketch is a rough outline, drawn lines, a snippet of code, a collection of comedic actions, or a brief, written overview [12] . The sketch across all domains is familiar in its brevity and fluid nature, inviting opinion - it is not a final option. In this way, sewing can perform a similar feat: by creating a fast, representative image borne of stitching, un-pickable, changeable, which invites further enquiry. Between sketching and sewing, there exist multiple entry points into the domain of $\mathrm{HCI}$ and the culture of Makerspaces. We examine first, the role of sketching in enquiry for $\mathrm{HCI}$, then the current space of stitching within this research space, before focusing on e-textile prototyping and sketching circuits.

\subsection{Sketching as Process in HCI}

Many HCI projects start with ideation and sketching, both in industry and academic circles [12]. Visual notes, outlines and iterations can be seen while individuals and teams work out the context and details of prototypes. Sketching is a useful tool for "trying out" ideas, for items or interfaces that do not yet exist [61,63], and is inherently speculative in nature $[60,65]$. Sketching is also a fact finding exercise, and imagery can be made, overlaid, annotated until the ideas become concrete [35]. The 'rough' nature of a sketch invites dialogue and can communicate intent [18]. Within computer science, the definition of a sketch is often loosely associated with the description of snippets of code, and these too, follow the same rules of scarcity and invitation to edit [14]. HCI as a field is broad, and the use of sketching is welcomed as a part of the design space of research. From basic pen and paper ideation [62], to sketching by robots [64], this simple process inspires and elucidates interactions.

\subsection{Stitching in $\mathrm{HCI}$}

Much like sketching, stitching is an ancient yet tangible process to create, mend and link materials. It has roots in purely functional items such as clothing and shelter, but can also be used to create artistic representations [25], communicate narrative or information [51], and, in the context of this work, support the making of functional, interactive prototypes [56]. The use of stitching in HCI has the advantage of being aesthetically pleasing which is of importance but also goes beyond this [41] - there is an ongoing dialogue between function and aesthetics. Stitching is playing multiple roles in designing and making computational products, as art, as craft, and also by bringing things together in a literal sense. As an example, Giles uses stitching as a process of creating multi-sensory prototypes with participants with visual impairments, as it offers a tangible method for creating lines and connecting spaces in electronic and textile media [17], likewise, Strohmayer and Meissner stitch quilted components together during co-creation, but also use the stitching to bind the wires into the fabric of their interactive 


\section{Oops! LED mistake}

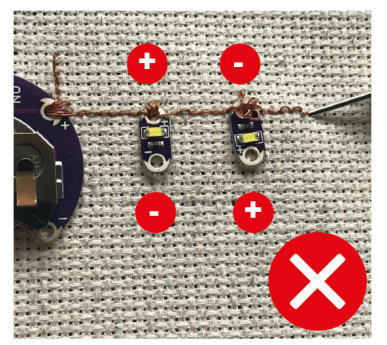

2. Pull thread to undo

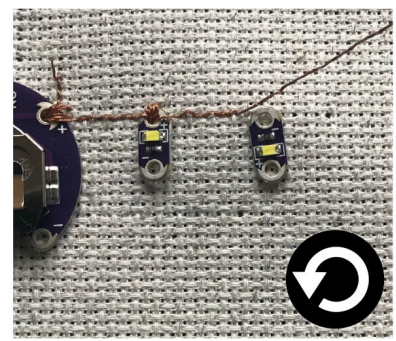

3. Continue punching - done!

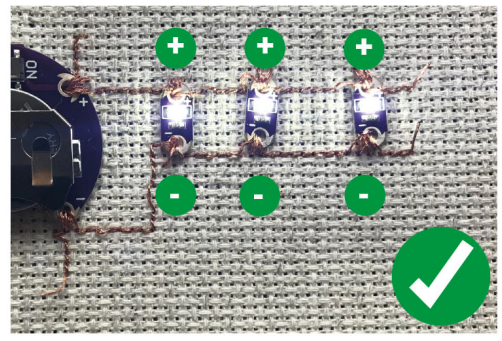

4. Pull thread to reuse

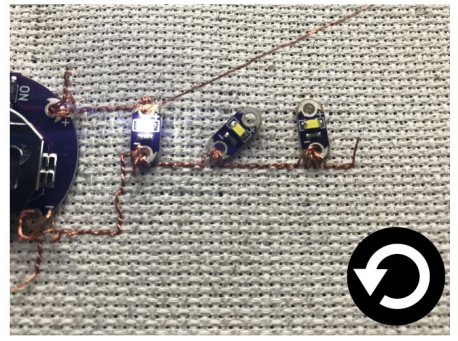

Figure 2: Punch-Sketching enables individuals to easily undo mistakes and reuse project components in future projects

Partnership Quilt [58]. In both examples, the stitching has both an aesthetic and functional purpose, creating electronic textiles which have multiple functions through the addition of digital components. Such e-textiles are becoming popular in education [16], makerspaces [57], and functional research (see next section), with continuing innovation to improve and inspire ways in which we work with the sewn line.

\subsection{E-textile Prototyping}

There are a wide variety of e-textile prototyping tools [50], but when prototyping with novices it is often easier to prototype concepts with toolkits with interchangeable parts. For example, LittleBits [2], an electronic toolkit with magnetic connections, has been used to co-design sneaker e-textile concepts such as MakerShoe [32]. Similar toolkits such as MakerWear [31] or BodyHub [46] attach directly to clothes or accessories for prototyping in place. Fabric toolkits such as Wearable Bits [29, 30], i CATch [43], Quilt Snaps [8], and Patchwork [4] connect with snaps and are better able to emulate what it feels like to wear or interact with e-textiles. The limitation of these interchangeable kits is their block-like form factor limits the creative expressiveness that is one of the major benefits of introducing individuals to e-textiles in the first place. The Arduino e-textile toolkit LilyPad $[6,7,10]$ has sewable components that have enabled new forms of creative expression and aesthetic interactions with soft objects, wearables, and furnishings. The use of thread connections is what allows prototypes to then live in the wild, as they functionally stay in place and allow for aesthetic decoration. Textile crafting approaches such as a Kit-of-No-Parts $[11,47]$, allow for creative expression to an even greater extent with techniques such as sewing, knitting, crocheting and felting. At the same time, because disassembly involves cutting or the use of a seam ripper (or e-seam ripper [48]), which is destructive to the threads, this makes it difficult and time-consuming to reuse project components.

\subsection{Sketching Circuits}

In contrast to the blocks approach of constructive assembly toolkits, sketching is the ability to draw in a freehand manner, and is useful for conveying ideas to others for discussion during the early stages of prototyping $[13,63]$. Several maker electronics toolkits have been created for "sketching" circuits on paper. The commercial BareConductive is a popular microcontroller and paint set that enables individuals to paint or stencil touch-capacitive interactions.
Similarly, pens with conductive silver ink have enabled individuals to draw circuits [55], and erasers like Circuit Eraser allow individuals to edit their drawings [42]. Several toolkits have developed around the expressive potential of conductive inks such as the StoryClip board [27] or TinyProgrammer [39] for illustrations, and a paper-based version of the LilyPad with magnetic connections for drawing computational sketchbooks [9]. Circuit sticker and copper tape kits $[23,52,53]$, such as those produced commercially by Chibitronics, are flat and enable circuit components to be almost flush with the paper for papercrafts. Larger scale "sketching" toolkits enable individuals to prototype interfaces for the body. Piezo-resistive kinesiology tape has the benefit of being flexible for removal and re-application [59]. Rapid Iron-On User Interfaces use tapes and patches with the benefit of having pre-made components that can easily be ironed in place, and ironed again to remove [34].

\section{PUNCH-SKETCHING E-TEXTILES}

In this paper, we propose Punch-Sketching e-textiles using a punch needle tool. A punch needle is a craft tool held like a drawing utensil that creates a continuous loop stitch when pushed through the fabric. To make each stitch, one punches the needle into the fabric, pulls it out, and glides it to the next punch location (Figure 1). This technique is commonly used in handmade rugs and uses the tension of the fabric weave to hold each stitch in place. Compared to other textile techniques, punch needle practitioners often describe their work in relation to other more freehand art methods. In her book Punch Needle, Khouunnaraj describes the method as "a textile art that has the ability to represent imagery, with a natural organic softness, and with an approach that seems like you are drawing with yarn” (p.4) [33]. Pearlman, the author of Modern Rug Hooking, describes her interest in punch needle in a similar manner, "I looked for a form of self-expression that was most similar to creating with paint" (preface) [45].

In addition to the ability to draw textiles, the benefit of this technique for prototyping e-textiles is that stitches will stay in place but are also easy to pull out and "undo" if pulled from a specific direction. Needle punch embroidery is of recent interest to HCI with machine fabrication of 3D embroidery [20], but has not been explored as a tool for manually sketching e-textile circuits or as a method of reusing threads. Notably, our approach is easy for other makerspaces and e-textile practitioners to reproduce since we use commercial punch needle embroidery tools, as well as other readily available craft supplies. Similar to A Kit-Of-No-Parts [47]and 
Crafting Technology [11], we look to different craft techniques within textile and fiber arts and explore how to adapt them to solve e-textile-specific problems.

\section{STUDY 1: UNDERSTANDING WITH FIBER ARTISTS}

To better understand how we engaged beginners in prototyping e-textiles using the punch needle technique, the tools and methods they use for their punch needle work, and how needle punch compares to other techniques, we began our research by interviewing two Fiber Artists (FA1 and FA2) who had recently started to use punch needle in their work. FA1 started working with punch needle two years ago but had also worked with embroidery, weaving and knitting. FA2 is a costume designer who had worked with a variety of stitching and embellishing techniques and had recently begun to use needle punch in decorative home items last year. In this study, we transcribed 2 hours of the video recording, extracted 8 codes and 2 main themes emphasizing the ease of use for beginners as well as the pictorial and representational nature of punch needle compared to other fiber arts. We performed inductive analysis with line-byline grounded codes for each quote. Codes were then grouped to develop narrative themes.

\subsection{Beginner Friendly}

Both fiber artists started with punch needle after seeing examples on social media, without buying toolkits or attending prior workshops. FA1 found that "just by looking at other people's art I could pick it up". For materials, they both built DIY frames. FA1 used "a canvas stretcher, and then ordered Monks cloth, stapled the cloth onto the stretcher to make a punch needle canvas". FA2 explained how they punch-needled: "[I] used a metal frame. I screwed them together, and then I sewed them by hand as tight as I could, and then I punched it." They both discussed making smaller works with embroidery hoops as frames, and FA1 also purchased a tension frame to avoid having to staple fabric and make holes. For needles, they both started off with thicker needles for yarn, before moving to thinner, adjustable needles for embroidery.

In comparison to other techniques, they found it "so much easier" [FA1]. FA1: "I found it super easy to pick up. It's just a regular stabbing motion". It was also quicker than other techniques. FA2 "never finished [needlepoint] embroidery because it's just too time intensive", but with punch needle she jumped right into completing a large project. FA2: "It was like, okay, let's go big. We're going to do a mountain view. It wasn't that difficult". They also found it very easy to correct errors. FA1: "Usually [the stitches] stay unless something snags and then I have to pull it through again. The good thing is that with punch needle you can literally just push the yarn back in, like even with a knitting needle you can just poke it back in to fix it. So, it's not like with knitting if you skip a stitch and you realize like 50 rows later." FA2 found it easy to make changes to her design when she ran out of a specific yarn expressing that "It was iterative." FA2 also stabilized loose threads by applying iron-on fusing material to the back of the image once she was done.

\subsection{Draw a Picture}

Both participants started using the technique to draw images with textiles. FA1: "I thought it was a really neat way to do something more representational" and "started drawing [images of] sculptures". FA2: "I wanted to do an embroidery with an image with a real scene. Doing this with normal stitching would have taken a very long time. So, I thought to do it with punch needle because it is really, really fast." In comparison to other techniques, punch needle was "much more flexible I think like just as an art form and as a fiber art" [FA1]. FA1: "I mean it's really versatile, you can make practical objects, you can make decorative things, you can make rugs, you can make wall art". The one limitation is that objects made with punch needle are difficult to wash due to the ability to pull the threads out. FA2: "I think it's cool for everything you maybe wash by hand once a year."

\section{STUDY 2: EXPLORING WITH BEGINNERS}

To better understand how Punch-Sketching e-textiles with a punch needle compares to sewing e-textiles with a sewing needle, we ran 2-hour individual beginner workshop sessions with 4 adult participants (P1-4) who have never used e-textiles before, using the tools and materials suggested during the interviews with fiber artist. We had a demo of a series and a parallel circuit (with LilyPad LEDs and a sewable battery pack) made with the punch needle and another that was sewn (Figure 3). We asked participants to replicate them once with sewing and another using the punch needle method. For each method they were asked to:

- Task 1: Create a straight line of conductive thread, a simple circuit, and a parallel circuit

- Task 2: Deconstruct the circuits for reuse.

We chose these activities to emulate those that would occur during beginner workshops. During the activities, we asked them to think aloud and discuss items they found easy or difficult. By running the study sessions in longer 2-hour periods, we were able to capture and discuss the micro-gestures and actions that participants take when doing both manual activities. The interviewer then asked clarifying questions based on each participant's feedback. In this study, we transcribed 8 hours of the video recording, extracted 18 codes and 4 main themes emphasizing benefits and limitations of Punch-Sketching and sewing e-textiles both for creation and deconstruction for re-use. We performed inductive analysis with line-by-line grounded codes for each quote including "activity event - participant state". For example, one of our codes was "needle punch - tension issues - frustration". These were then used to develop themes based on how participants made sense of and interacted with the materials.

\subsection{Juggling Peripheral Attention}

The participants enjoyed the process of lighting up their circuits and they all described the process as "fun". At the same time our beginners found that there were a lot of things that they had to be aware of while building e-textile circuits with both techniques (Figure 3). The positive ones related to "what it looked like and how the pieces were connected" [P4], and the creativity of making their "own style of circuit" [P2], but anything else that needed their 


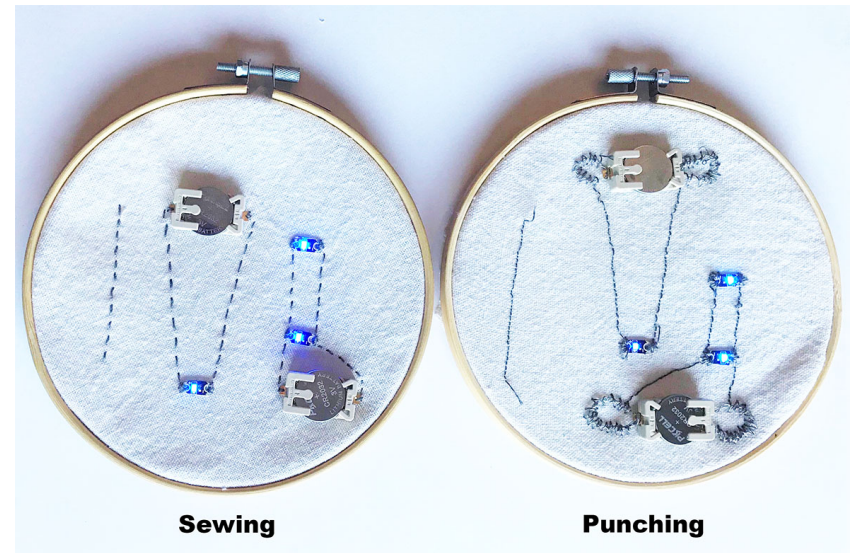

Figure 3: The activity examples participants were shown before starting (left) sewing e-textiles with a sewing needle (right) Punch-Sketching e-textiles with a needle punch tool. It took participants an average of 45.40 minutes to sew their circuits, 11.42 minutes to take apart the sewing whereas punching took on average 28.12 minutes and 1.44 minutes to take apart their punched circuits

attention was considered distracting. We used off-the-shelf HighFlex 7-ply Karl Grimm silver-plated conductive thread. Participants found that this conductive thread "takes some getting used to" [P1], and they frequently described it as "stiffer", "thicker", "frays", "kinks" and "catches easily" [P1,2,3,4] compared to regular thread.

During sewing, these features of the thread came into focus. All participants found that the thread "has a tendency to get stuck" [P2] and they had to correct accidental knots that occurred on the back. Even after noticing this issue on one knot, it would often reoccur when their focus shifted to other parts of the circuit design. As P4 described sewing with conductive thread, "I think there's an element of mindfulness to this one, I can't trust that it is pulled through all the way unless I check it". This led to all participants constantly "checking on both sides" [P4] and "propping [a] finger in the middle" [P2] to slow down the thread and avoid knots.

In contrast, the thread's tendency to kink was seen as a positive during punching since it "remembers" [P2] and holds its shape after being punched. P1: "You're just punching it. You don't have to go through looking at both sides of the material." These kinks helped to hold the punched stitches in place, but there were other peripheral elements that sometimes distracted participants while punching circuits. With both techniques the participants used embroidery hoops, but during punching the hoops became looser as they punched, which affected the stitches. Those who recognized that "it just needed more tension" [P01] would tighten the hoop and continue stitching, but those who did not notice would become frustrated with how "loose the stitches are" [P03].

As $\mathrm{P} 4$ increasingly focused on the design of their circuit, the hoop tension "became peripheral, and I probably would not have noticed that distinction between how it was hanging, and related that with particular difficulties". Another tension issue was that some participants accidentally held the hanging thread inside their tripod grasp of the punch needle, which meant that they would accidentally pull each stitch out right after they punched it. These two findings suggest that e-textile Punch-Sketching can benefit from high-flex quality conductive thread, stronger fabric frames and/or a spool on the punch needle to hold threads up and out of the way, so that participants can focus on what is important - their design.
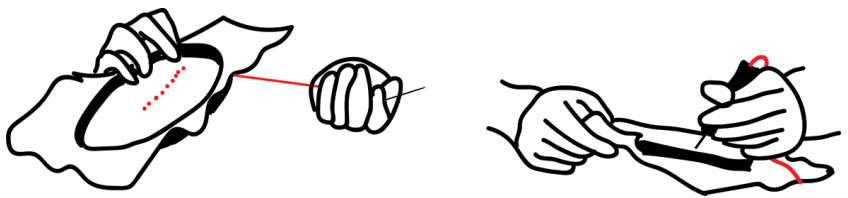

Figure 4: (left) flipping the embroidery hoop over to check for knots, (right) accidentally gripping the thread in the punch needle grasp.

\subsection{Wasteful Sewing}

After creating each circuit, we asked participants how they would take out the circuit to reuse items for the next activity. During 'sewing' some tried initially to re-use the thread but described it as "tedious" [P1] and "an unnecessary evil" [P2]. In the end, all participants decided to snip the components out with scissors and decided to save "none of the thread" [P3]. Altogether, the knots, short length, and fraying of the thread kept participants from wanting to re-use it. As P2 described the thread's journey during sewing, "It gets cut, first of all, '[be]cause you're putting it on your needle, and then when you're sewing it, because it's like an aluminum tinsel, it makes all these weird turns, [and] there are a lot of loose fibers where it breaks." After pulling the threads out participants had "little wee bits" [P2] and "a tangled mess of wire" [P4]. Participants also felt negatively about not being able to re-use the threads calling it "a shame" [P1] and P4 saying "it seems wasteful even though I know it is being taken apart to reuse items."

In contrast, after the 'punching' activity, all participants said that they would be able to re-use the threads "for the right activity" [P04]. As P2 describes the process "I'd just pull the thread out. I could reuse that one, and that's a long piece." The threads that were retrieved in this method were longer partially because the punch needle creates loops, but also because the former sewing method did not support disassembly. Despite the possibility of being a drawback, all participants mentioned the length of the threads -incase of punching- as an advantage and the reason that it could be reused. They also described the retrieval process as "easy". The other circuit elements including the LEDs, battery packs, and embroidery hoops were all seen as reusable in both activities.

\subsection{Sew for Permanence}

Instead of preferring one technique to the other, participants viewed punching and sewing as useful for different purposes. Participants described sewing as a permanent process with "knots" [P2,P3,P4] and "stay stitches" [P1] that were used for "security", "longevity", and to "lock it [...] so it's not going anywhere" [P3]. As P1 states "I usually stitch something so that it doesn't come out". They also felt that the e-textile components were more secure when sewn in place. 
All participants also discussed how sewing circuits required preparation. It required "foresight and planning [for] where everything was going in advance, because once [a] stitch was in there, going back is not really a possibility" [P4]. Before sewing, all participants said it was important to "measure" and "gauge the distance" [P1] before cutting the thread. Most of the errors participants made were things that they were not able to fix without starting over. Two participants focused on the thread explaining: "I underestimated how much thread I needed" [P2] and "what we are learning from this is that I need more thread than I think" [P4]. The other two focused on the placement of the circuits: "I wasted some material" [P1] and "I don't think I left enough room to do all three experiments" [P3]. The combination of sewing, knotting and placement problems created what $\mathrm{P} 4$ called "the multiple thread danger zone in which I have no thread."

\subsection{Punching for Prototyping}

In contrast to sewing, punching circuits enabled participants to work on the fly and change direction as needed. Participants described punching as "a snap" [P1], "quick" [P2], and "fast" [P3]. P4: "It was very satisfying do a quick line and see it come together at a relatively rapid pace." Two participants [P1 and P4] emphasized how it was easier than going "in and out" with sewing. Mid-way through punching in two parallel LEDs, $\mathrm{P} 4$ decided to try adding 2 more "because the thread here is just continuous, I don't feel like I'm at the end of the thread, and I just keep going". Participants described punched circuits as very easy to undo by pulling. P2: "It's kinda fun, if you mess it up you just pull it out and try again". You could also redirect a line in progress. P4 upon realizing an error, wound up her stitches and then started punching in the other direction with the same thread. By doing so she was "able to keep what I had done with the LED and then just redirect it. Whereas I don't think I could have done that redirection with sewing".

Based on the ability to quickly undo and redo, all participants recommended punching for "prototyping", "experimenting", "testing", "trial and error", and "hands-on learners", and then recommended using the more permanent nature of sewing for the final version or "finished product" [P4]. P3: "I could use this to quickly throw up a quick circuit just to make sure it works, and then pull the thread and stitch it more permanently." P1 compared the punch tool to a basting stitch, which is used to hold fabric temporarily in place: "It's so much less time consuming and it would be great for basting. For trying to figure out stuff, for experimenting. And then use sewing when you've got your plan made." Punch needle was good for coming up with a plan, "in terms of creativity, to add or to improvise" [P4]. Participants did not choose between punching or sewing, they recommended punching then sewing.

\section{STUDY 3: UTILIZING WITH OCCUPATIONAL THERAPISTS}

Occupational therapists evaluate tasks, tools, and technologies from a holistic perspective that includes the person, the environment, and the activity [1]. This gives them a unique understanding of how tools are understood by the body's senses and what factors we need to consider when comparing them. In previous work, interviews and collaborations with occupational therapists have led to a better understanding of how to make tools easier to use for diverse populations $[1,28]$, and in the context of a makerspace these insights can give facilitators some ideas for items to have on hand. Moreover, prior work highlighted the relationship between occupational therapy and 'making' [24] and suggested the great value of hands-on methods and craft.

Therefore, after running Study 2, we opted for evaluating our punching method further and video interviewed 10 occupational therapists (OT1-10) individually to discuss the results and to better understand the differences between the two methods. Interviews started with a demonstration of each technique (sewing and punch needle for e-textiles) and then participants were asked to compare the two based on their area of expertise within OTs. We video recorded and transcribed the interview sessions, each lasting between 30-45 minutes, and used Thematic Analysis to capture the codes and themes. We performed inductive analysis with line-byline grounded codes for each quote. Codes were then grouped into themes. In this study, we transcribed 8 hours of the video recording, extracted 14 codes and 3 main themes emphasizing benefits and limitations of Punch-Sketching in terms of being a useful learning tool, a support for dynamic grasp and its adaptability as a method.

\subsection{Punch Needle as a Learning Tool}

All the OTs referenced using 'task analysis' to break down the steps and context of a task. Several participants described the punch needle as an intermediate tool between learning how to draw using drawing utensils and sewing with a needle. OT3: "Kids are colouring, so if you have someone who is not understanding the sewing needle this is a good way to introduce the process. If it is too hard, they're going to lose motivation. So, you have to find that fit for them to be able to feel accomplished and actually working on something". They also discussed how the punch needle is easier for cognitive sequencing of the task. Instead of threading a new sewing needle for each circuit trace, with the punch needle users could load the thread at the beginning or it could be "facilitators who are doing more of that versus the users" [OT4]. Being able to build circuits on the fly without having to preplan the thread length would also be beneficial for individuals with "reduced impulse control and planning" [OT4]. For both sewing and punching, the OTs recommended using stencils, pipe cleaners, or taped guides, "especially for kids that need a little bit more guidance" [OT7]. The speed of punching would also be useful for individuals who fatigue easily.

All the OTs also compared the visual element of using a sewing needle and a punch needle. OT7: "When it comes to sewing vision is key, especially those tiny little things." They discussed how the punch needle would be easier to see and would have the benefit of focusing "your visual field on the front of the canvas" [OT1]. They also discussed the benefit of adding contrasting colours for the needle, thread, and fabric. OT3: "Whenever you have low vision you just want big colours for contrast". OT6: "If your thread is close to the colour of the fabric they may have more difficulty". In terms of learning the technique they also discussed the added auditory feedback of the sound of the needle punching through the fabric. 


\subsection{Supporting Diverse Grasps}

All OT participants discussed the differences in how you would hold and stitch with a sewing needle and a punch needle, and the grasp patterns involved (Figure 5). They emphasized that "a smaller object requires a more dynamic grasp" [OT3]. The OTs described sewing with a needle as a two-finger pincer grasp that uses fine motor control where everything from your wrist, to small "finger movements"[OT1], to the "the very tip of the finger" [OT5] is involved in controlling the needle. OT1: "When you're usually doing needlework it's more of a wrist and finger movement". In contrast, the OTs described the punch needle as having more possibilities for a larger "gross movement, as in movement of the shoulder as a unit" [OT1] or the "elbow joint" [OT4]. OT5: "If they can't move their wrist enough, they can just do the up and down motion" which requires "less rotation of the wrist and less dexterity" [OT1]. In terms of the grasp patterns, the punch needle was also described as having more flexibility for a variety of grasp patterns including "holding it like a marker" [OT2] with a tripod grasp, or a fisted palmar grasp.
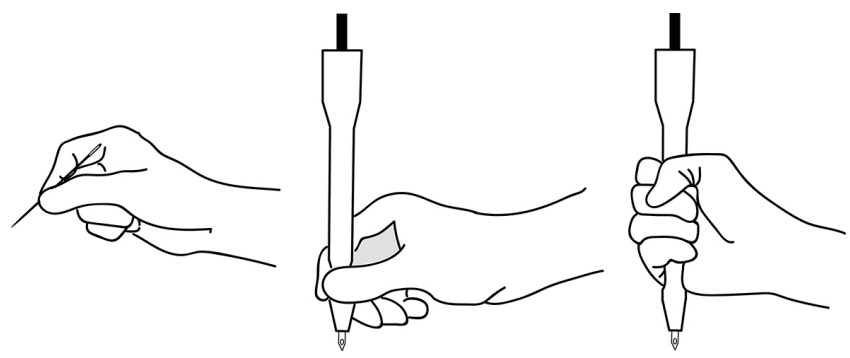

Figure 5: The different grasp patterns discussed by the OTs (left) two-finger pincer grasp with a sewing needle, (middle) three-finger tripod grasp with the punch needle, (right) palmar fist grasp with the punch needle

The OTs also discussed the benefits of designing e-textile tasks to be unimanual (one-handed) instead of bilateral (two-handed). OT5: "Besides the hand that is doing the actual sewing, we would have to consider what is being sewn. Like is it flat, is it held by the other hand, is the other hand involved or is it stabilized by another surface?" They described e-textile sewing as a bi-lateral activity because individuals hold the needle in one hand and the fabric in the other. Even when the fabric is stabilized, individuals often use one hand to push the needle through the fabric and the other to pull it from the other side. In contrast, the punch needle only needs to be accessed from 1 side of the fabric so "there's more adaptations that you can make" [OT1]. OT4: "The product could be stabilized by something and then it'd be easier to control with just one hand". They recommended DIY adaptions such as easels, wedges, as well as commercial adaptive frames. These insights emphasize the importance of having a secure frame for punch needle activities.

\subsection{For Adaptions, Bigger is Better}

Sewing needles move through the fabric and therefore need to remain relatively thin, though there are plastic embroidery needles that are slightly larger and easier to use. In contrast, the OTs emphasized that the punch needle "gives you more surface area to grasp" [OT7] and offers more adaptation opportunities. The shift from a thin needle to a wider handle was already a "big adaption" [OT3], but the punch needle also offers "more ways to modify it than the regular needle, cause sometimes an individual might wear a splint or an armbrace, and as an OT we could attach a tool to support that if they don't have that grasp" [OT5]. OTs recommended creating "a built-up handle" [OT1] as well as "using a longer punch needle so that it's easier to hold with different grasp patterns" [OT2]. These additional handles could be made through a variety of DIY methods including foam tubing, duct tape, clay, moldable glue (Sugru), play dough, pipe cleaners or pencil grips. The OTs also emphasized how these DIY adaptations are very common when supporting clients on the fly, for example OT8 said "My glue gun is my best friend".

Another adaptation the OTs discussed was the use of the universal cuffs (e.g. Figure 6e) that individuals might already use for drawing and eating utensils. OT1: "For the pushing and pulling, you could attach it to a cuff for someone who can't hold it." OT9: "So you could slide a tool into [the cuff] so [they] wouldn't even have to hold it". Because of the large handle and the punching of the needle through one side of the fabric, several OTs mentioned that the punch needle could help bring e-textile sewing to those who would not find the activity safe with regular needles. OT1, OT3 and OT5 discussed opportunities for mouthpieces: "If it's on an easel 1 could see somebody who doesn't have any arm function using it with their mouth to then poke forward with their neck. So, besides their hand, what are the other options, and could it be applied to other body parts" [OT5]. These diverse insights and adaptation opportunities give makerspaces options for the kinds of items they might have on hand to help make e-textile needle punching more accessible.

\section{TOOLKIT RECOMMENDATIONS}

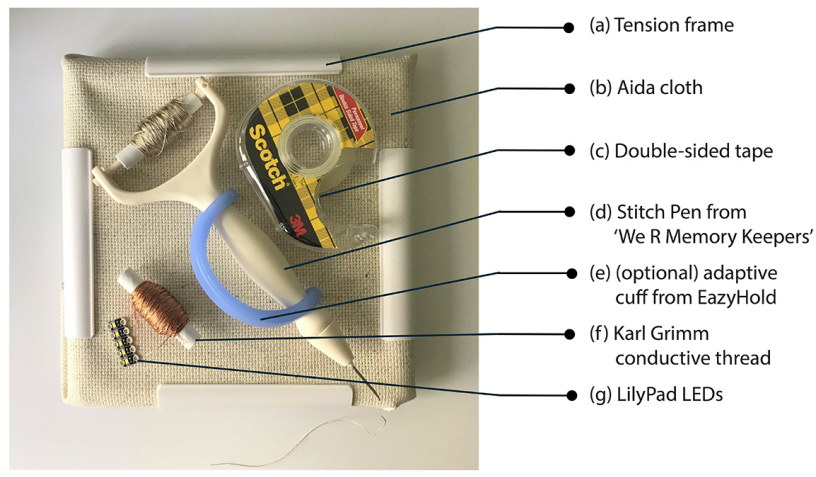

Figure 6: Suggested Punch-Sketching e-textiles toolkit with (a) tension frame, (b) Aida cloth, (c) double-sided tape, (d) Stitch Pen from 'We R Memory Keepers', (e) (optional) EazyHold adaptive cuff, (f) Karl Grimm conductive thread, (g) LilyPad components

Punch-Sketching combines the aesthetic aspects of fiber arts with the functional nature of creating circuits. As such, there are some 
requirements that are unique to working with e-textiles compared with working with regular punch needle tools and materials, such as the stitch tightness around electronic components and connections. Based on our study sessions and interviews we recommend the following toolkit (Figure 6):

- Needle: Our beginner participants tended to hold the yarn in their grasp of the punch needle, which caused them to accidentally pull out stitches. Our OT participants also discussed the benefits a tool with both a wide and long handle. We therefore chose the We R Memory Keepers Stitch Pen for its handle, as well as the ability to hold thread in a spool and therefore out of the way. Note that this pen is made for cutting through paper, so the needle needs to be sanded a little with a nail file to smooth it for use with fabric. Sand it until the edges are smooth when felt against your finger.

- Fabric: Aida cloth is commonly used in embroidery and its larger holes enable participants to re-use the same fabric for different exercises.

- Frame: Though embroidery hoops are commonly used for punch needle, we found that with beginners the need to adjust the tension was a distraction and if they forgot about it their stitches were loose. As a result, we suggest instead using a tension frame which will hold the fabric in place. Attaching optional stick dots to the bottom of the frame will grip the frame to the table surface and make it easier to use the punch needle with one hand.

- Thread: All types of embroidery threads can be used for punch needle, but the high-flex 7-ply Karl Grimm copper or silver-plated thread kinks and "remembers" its shape which holds it in place better than other threads. The OTs recommend contrasting colours for populations with low vision.

- Components: Not all e-textile components can be used with punch needle. We found that components with larger through holes (approx. 3mm or more) such as the LilyPad enabled participants to do several zigzag stitches along the through hole, which kept the components in place even when held upside down. Toolkits especially for Punch-Sketching would benefit from even larger through holes to enable more zig zag stitches.

- Accessories: To support unimanual tool use we recommend using double sided tape on the back of components to temporarily hold components in place while individuals make their punch needle stitches.

It is also important to note that the fabric, needle, and fiber (thread or yarn type) are connected. If these three items are matched correctly you will not need to add any adhesives on the back of your project to keep it in place. If your threads fall out, then one of these items is not matching the others. As one item scales up or down the others need to too. For example, if you are using a thicker conductive yarn, you will need a wider needle (such as an Oxford Needle), and a fabric with a looser weave (such as Monk's Cloth). If you want to use thinner threads (such as a single thread), you would need a thinner needle (such as Igolochkoy ${ }^{\mathrm{TM}}$ needle), and a fabric with a tighter weave.

\section{APPLICATIONS}

The technique of Punch-Sketching e-textiles provides new opportunities for re-usable e-crafting, accessible making, soft interfaces, and iterative prototyping.

\subsection{Re-useable E-Crafting}

Punch-Sketching brings e-textiles toolkits one step closer to a constructive assembly that is both re-usable, and creatively expressive (Figure 7). Though our participants used conductive thread and e-textile components, the technique can also be expanded and combined with other non-conductive materials such as colourful embroidery floss and yarns of varying widths. Our aim is that individuals can explore and learn about the creative potentials of e-textiles with all the flexibility provided by using thread, while still being able to work iteratively to easily undo mistakes and redo stitches. These features provide new opportunities for education and quickly learning through errors rather than getting frustrated by them.

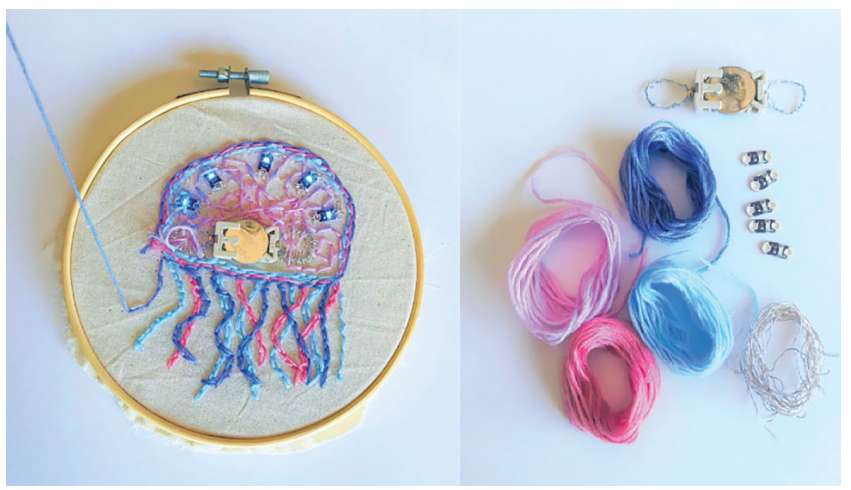

Figure 7: An e-textile jellyfish sketch made with the punch needle and being pulled out by one thread (left), the threads wrapped up for reuse after pulling them out (right).

\subsection{Accessible Making and Crafting Tools}

The movement from a small sewing needle to a larger punch needle provides an easier grasp and turns a bilateral task into a unimanual task. This provides accessibility opportunities for people with limited hand dexterity or mobility impairments, but there are also a wide variety of adaptions that can be added based on our discussions with OTs. Also, the benefit of using a tool with a tripod grip is that individuals with disabilities can re-use assistive tools they might already have for writing, drawing, and utensil use (such as the EazyHold grip). For example, occupational therapists often recommend assistive tools such as universal cuffs or built up foam grips. Instead of focusing on one specific disability, our OT interviews aim to provide makerspaces with ideas of accessories and craft supplies that they can have on hand to support the diverse populations that might walk through their doors.

\subsection{Fuzzy or Furry Interfaces}

In this paper we present using the punch needle technique with the loops facing down, but the original purpose of the punch needle within fiber arts is for making hooked rugs and textured surfaces. Combined with Punch-Sketching e-textiles, the loops created by the punch needle can also be utilized for fuzzy or furry interfaces (Figure 8). To do so, individuals can sketch on the back using conductive threads and yarns, and then flip their work over to present 
the loops side up. This technique can also be used with conductive yarns, with longer needles providing longer loops. This looping technique provides new aesthetic and design opportunities for individuals to draw friendlier and fuzzier interfaces, similar to Margaret Orth's tufted interfaces [44].

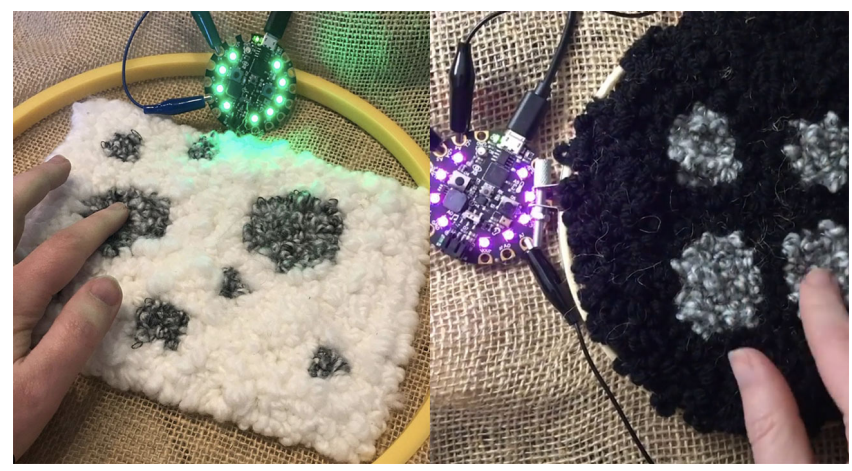

Figure 8: two furry interfaces for controlling mood lighting made with punch needle

\subsection{Prototyping in 3D}

For e-textile practitioners and as well as multidisciplinary teams from other fields, such as fashion and interior designers, PunchSketching e-textiles can enable them to quickly sketch out concepts and test out ideas in a free-hand manner (Figure 9). In this way the tool can extend upon the practices of manually sketching that these fields already have, with the added opportunity of testing out ideas in-situ. For creatives with practice with the tool, the punch needle enables them to sketch in space without using a tension frame and instead holding the fabric between their fingers.
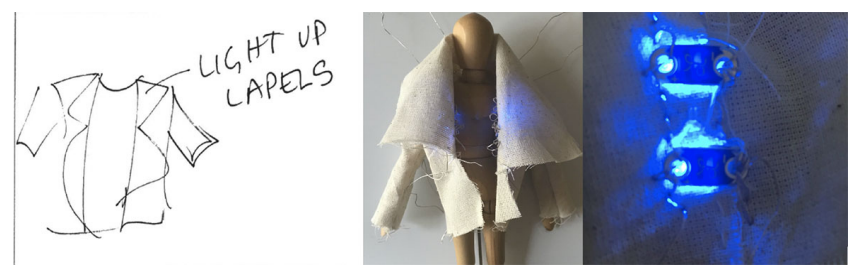

Figure 9: With practice practitioners can use the punch needle to quickly prototype $3 \mathrm{D}$ objects in-situ without a tension frame. In this example, a wearable jacket concept goes from sketch (left), to quick mock-up using the punch needle (middle), with a close up of the punched stitches (right)

\section{DISCUSSION}

We believe that the technique of adapting the punch needle to sketch sustainable, adaptable circuits, has potential to be extended further in e-textiles research. So far we have demonstrated that the Punch-Sketching technique has three major benefits: first being that the technique is easy to reproduce and accessible to makers and researchers in that it uses commercial tools and craft supplies; second, in that the sustainability of the technique is embedded in the fact that materials can be re-used several times - with the thread being pulled out and made available for subsequent workshops; and, third, the stitched line is easy to do/undo - the ability to edit along the way takes the frustration out of prototyping and getting things wrong the first time. The fact that the punch-needle technique is already known to those who stitch and craft means that by using this tool we allow people to leverage existing skillsets, which may also have the benefit of inviting new makers into the space of e-textiles.

\subsection{Sketching E-textiles as Process}

Our method has demonstrated the efficacy of the punch-needle to mirror the swift ease with which we can sketch a concept with a pencil and paper, much as Khouunnaraj [33] likened the technique to be most similar to drawing with yarn. Sketches should not be laborious, slow works, they need to work concurrently with the thought process of the individual, they should allow for further creation after the fact. Punch-Sketching e-textiles also bridges the gap between stylus based sketching and rapid-prototyping - being part of both processes.

When we are familiar with tools, we are more likely to feel able to engage in a situation, even if the context is novel [60] - and further, we can also use familiar tools without extensive training, such as mapping gestures associated with the measuring tape to the digital space [19], or digital stylus with extra interactions [22, 38]. Our use of the punch-needle, a popular method of stitching quickly and freely, may open the e-textiles space to users who are already familiar with stitching, but are unfamiliar with the e-textiles space. Current works in e-textile tool development have looked to adapt existing items, for example, Posch's E-textiler's Tape tool [49], adding connectivity in existing needlecraft tools such as crochet hooks [48], and even creating wearable components to assist with other tools [21] - and this shows that there is the potential for developing brand new methods for testing and creating e-textiles. However, there is also the potential to identify, and leverage existing tools - such as the punch-needle - to invite ease into e-textiles methods. The future of tools and methods in e-textiles is thus likely to be a hybrid space.

\subsection{Sustainability in E-textiles}

Sustainability is a wicked problem [5] in that the systems involved are so complex and are so rarely fully visible to us that we can only slowly chip away at solutions. In e-textiles, and in textiles and electronics in general, this complexity is heightened through opaque manufacturing systems. Similar to the work of Unfabricate [66], we aim to address sustainability through designing for disassembly and reuse. With Punch-Sketching e-textiles our sustainability aims were narrow, to enable makerspaces to reuse e-textile materials across workshops, and many problems within the manufacturing of e-textiles remain. At the same time, the Punch-Sketching concept of making, un-making and re-making could be expanded to customizable products. For example, one could easily imagine a tote bag made of a punchable material where the design could be taken out and updated whenever someone wants a new look. The 
ability to erase means we never need to get rid of something, we can simply edit or update it until it actually wears out.

\subsection{Ergonomics and Accessibility}

Whilst Punch-Sketching e-textiles is accessible in terms of availability and cost, it also has the potential to be adapted for users with different needs. We intend to examine whether existing commercially available grips for pens and other tools might be adapted for use with the punch-needle. This would provide wider access to the technique and hopefully allow those who may not currently have the ability to engage with e-textile circuitry the opportunity to create their own prototypes. In terms of ease of use, the tool could also be adapted to be integrated with a wearable meter for testing and debugging circuits in real time, such as that investigated by Hill et al. [21] - meaning testing was hands-free and not a subsequent act. Combined with the ability to easily unpick and re-sew lines, this also has the advantage of speeding up the making process - in this way we can physically "CTRL-Z" and undo, much as we would whilst using screen based media.

\subsection{Reflection}

Although we position our Punch-Sketching e-textiles technique in the creation of functional circuitry, there also lies the potential to bridge the gap between function and aesthetics. By this we mean that the functional sewn line might also serve a dual purpose to be both circuitry and representational - the art being of and in itself the link. Stitching as a notion also means to bring things together and bind them, and makerspaces bring people together in mutual interests, and educate. Our sketching technique is but one method of creating e-textile prototypes, but its nature means that it may have possibilities to extend the reach of workshops and educational spaces by making the learning and doing much easier. The ability to adapt existing analogue tools to be part of the creation of physical, digital prototypes and artworks means that traditional crafting techniques will persist and have a life in the on-going digital world. In this way, our technique is as much part of preserving heritage as striving for innovation.

\subsection{Limitations \& Future Work}

Though there are broad applications for Punch-Sketching prototypes, there are several limitations to the technique. As mentioned earlier, the fabric, needle, and fiber (thread or yarn type) are connected, so an individual needle will not work with all fabrics, and practitioners often have several needles for working with different materials. In contrast, a sewing needle will work with most fabrics. The technique is also not useful for prototyping concepts that utilize or play with the resistance of threads, such as soft speakers [40] that have to be to be exactly 4,8 or $12 \mathrm{Ohms}$ to function, because the length of a prototyped punched trace will be approximately 3 times longer than the final sewn trace, and will therefore have a different resistance.

Further research with more participants is needed for quantitative data on the technique as well as for the long-term use of Punch-Sketching, and how well the technique withstands use, and re-use. In this project we aimed for using commercial products, but further work is also needed on how we could design components specifically for punching. Finally, though we interviewed occupational therapists on the design of the tool, further testing with individuals with disabilities is needed to fully evaluate the accessibility of the tool.

\section{CONCLUSION}

In this paper, we introduce the method of Punch-Sketching etextiles with a punch needle, which enables individuals to quickly undo and redo stitches as well as re-use materials for future projects. To better understand the use of punch needle as an accessible makerspace crafting tool we ran 3 studies with a total of 16 participants. During these studies, we conducted interviews with fiber artists (2), workshops with e-textile beginners (4), and interviews with occupational therapists (10). Based on insights gained, the paper provides recommendations for how to use this crafting method in makerspace settings and we discuss its applications for re-usable crafting, fuzzy interfaces, and prototyping e-textiles. Our findings also suggest the value of Punch-Sketching for accessible making and engaging them in inclusive design ideation. Our aim is for beginners and practitioners to leverage the capabilities of fiber arts tools in new ways to solve current e-textile prototyping barriers.

\section{ACKNOWLEDGMENTS}

We would like to thank all our participants who generously contributed their time and ideas to this study. We would also like to thank our reviewers for their insights that helped to improve this work. This work was supported and funded by the National Sciences and Engineering Research Council of Canada (NSERC) through a Discovery grant (2017-06300), a Discovery Accelerator Supplement (2017-507935), by the Ministry of Ontario through an Early Researcher Award (ER15-11-101), and a Mitacs Research Training Award. The views expressed in the publication are the views of the Institution and do not necessarily reflect those of the Province.

\section{REFERENCES}

[1] Leila Aflatoony and Su Jin (Susan) Lee. 2020. AT makers: a multidisciplinary approach to co-designing assistive technologies by co-optimizing expert knowledge. In Proceedings of the 16th Participatory Design Conference 2020-Participation (s) Otherwise-Volume 2. 128-132. https://doi.org/10.1145/3384772.3385158

[2] Ayah Bdeir. 2009. Electronics as material: littleBits. In Proceedings of the 3rd International Conference on Tangible and Embedded Interaction. 397-400. https: //doi.org/10.1145/2148131.2148220

[3] Eli Blevis. 2007. Sustainable interaction design: invention \& disposal, renewal $\&$ reuse. In Proceedings of the SIGCHI conference on Human factors in computing systems. 503-512. https://doi.org/10.1145/1240624.1240705

[4] Amanda Boone, Eileen Rivera, and Jacob Wolf. 2018. Patchwork: an expressive e-textile construction kit. In Proceedings of the 17th ACM Conference on Interaction Design and Children. 529-532. https://doi.org/10.1145/3202185.3210770

[5] Richard Buchanan. 1992. Wicked problems in design thinking. Design issues 8, 2 (1992), 5-21.

[6] Leah Buechley. 2006. A construction kit for electronic textiles. In 2006 10th IEEE international symposium on wearable computers. IEEE, 83-90. https://doi.org/10. 1109/ISWC.2006.286348

[7] Leah Buechley, Mike Eisenberg, Jaime Catchen, and Ali Crockett. 2008. The LilyPad Arduino: using computational textiles to investigate engagement, aesthetics, and diversity in computer science education. In Proceedings of the SIGCHI conference on Human factors in computing systems. 423-432. https: //doi.org/10.1145/1357054.1357123

[8] Leah Buechley, Nwanua Elumeze, Camille Dodson, and Michael Eisenberg. 2005. Quilt snaps: A fabric based computational construction kit. In IEEE International Workshop on Wireless and Mobile Technologies in Education (WMTE'05). IEEE, 3-pp. https://doi.org/10.1109/WMTE.2005.55 
[9] Leah Buechley, Sue Hendrix, and Mike Eisenberg. 2009. Paints, paper, and programs: first steps toward the computational sketchbook. In Proceedings of the 3rd International Conference on Tangible and Embedded Interaction. 9-12. https://doi.org/10.1145/1517664.1517670

[10] Leah Buechley and Benjamin Mako Hill. 2010. LilyPad in the wild: how hardware's long tail is supporting new engineering and design communities. In Proceedings of the 8th ACM conference on designing interactive systems. 199-207. https: //doi.org/10.1145/1858171.1858206

[11] Leah Buechley and Hannah Perner-Wilson. 2012. Crafting technology: Reimag ining the processes, materials, and cultures of electronics. ACM Transactions on Computer-Human Interaction (TOCHI) 19, 3 (2012), 1-21. https://doi.org/10.1145/ 2362364.2362369

[12] Bill Buxton. 2010. Sketching user experiences: getting the design right and the right design. Morgan kaufmann.

[13] Brock Craft and Paul Cairns. 2009. Sketching sketching: outlines of a collaborative design method. People and Computers XXIII Celebrating People and Technology (2009), 65-72.

[14] Kathryn Cunningham, Sarah Blanchard, Barbara Ericson, and Mark Guzdial. 2017. Using tracing and sketching to solve programming problems: replicating and extending an analysis of what students draw. In Proceedings of the 2017 ACM Conference on International Computing Education Research. 164-172. https: //doi.org/10.1145/3105726.3106190

[15] Kristin N Dew and Daniela K Rosner. 2019. Designing with waste: A situated inquiry into the material excess of making. In Proceedings of the 2019 on Designing Interactive Systems Conference. 1307-1319. https://doi.org/10.1145/3322276.3322320

[16] Deborah Ann Fields, Yasmin Kafai, Tomoko Nakajima, Joanna Goode, and Jane Margolis. 2018. Putting making into high school computer science classrooms Promoting equity in teaching and learning with electronic textiles in exploring computer science. Equity \& Excellence in Education 51, 1 (2018), 21-35.

[17] Emilie Giles, Janet Van der Linden, and Marian Petre. 2018. Weaving lighthouses and stitching stories: Blind and visually impaired people designing e-textiles. In Proceedings of the 2018 CHI Conference on Human Factors in Computing Systems. 1-12. https://doi.org/10.1145/3173574.3174044

[18] Saul Greenberg, Sheelagh Carpendale, Nicolai Marquardt, and Bill Buxton. 2011 Sketching user experiences: The workbook. Elsevier.

[19] Chris Harrison, Robert Xiao, Julia Schwarz, and Scott E Hudson. 2014. TouchTools: leveraging familiarity and skill with physical tools to augment touch interaction. In Proceedings of the SIGCHI Conference on Human Factors in Computing Systems. 2913-2916. https://doi.org/10.1145/2556288.2557012

[20] Shiqing He and Eytan Adar. 2020. Plotting with Thread: Fabricating Delicate Punch Needle Embroidery with XY Plotters. In Proceedings of the 2020 ACM Designing Interactive Systems Conference. 1047-1057. https://doi.org/10.1145/ 3357236.3395540

[21] Chris Hill, Michael Schneider, and Mark Gross. 2020. A Wearable Meter That Actively Monitors the Continuity of E-Textile Circuits as They Are Sewn. In FabLearn'20.

[22] Ken Hinckley, Michel Pahud, Hrvoje Benko, Pourang Irani, François Guimbretière, Marcel Gavriliu, Xiang'Anthony' Chen, Fabrice Matulic, William Buxton, and Andrew Wilson. 2014. Sensing techniques for tablet+ stylus interaction. In Proceedings of the 27th annual ACM symposium on User interface software and technology. 605-614. https://doi.org/10.1145/2642918.2647379

[23] Steve Hodges, Nicolas Villar, Nicholas Chen, Tushar Chugh, Jie Qi, Diana Nowacka, and Yoshihiro Kawahara. 2014. Circuit stickers: peel-and-stick construction of interactive electronic prototypes. In Proceedings of the SIGCHI Conference on Human Factors in Computing Systems. 1743-1746. https://doi.org/10.1145/ 2556288.2557150

[24] Megan Hofmann, Kristin Williams, Toni Kaplan, Stephanie Valencia, Gabriella Hann, Scott E Hudson, Jennifer Mankoff, and Patrick Carrington. 2019. " Occupational Therapy is Making" Clinical Rapid Prototyping and Digital Fabrication. In Proceedings of the 2019 CHI Conference on Human Factors in Computing Systems. 1-13. https://doi.org/10.1145/3290605.3300544

[25] Shu Hung and Joseph Magliaro. 2007. By hand: The use of craft in contemporary art. Princeton Architectural Press.

[26] Hiroshi Ishii. 2008. Tangible bits: beyond pixels. In Proceedings of the 2nd in ternational conference on Tangible and embedded interaction. $\mathrm{xv}-\mathrm{xxv} . \mathrm{https}$ : //doi.org/10.1145/1347390.1347392

[27] Sam Jacoby and Leah Buechley. 2013. Drawing the electric: storytelling with conductive ink. In Proceedings of the 12th International Conference on Interaction Design and Children. 265-268. https://doi.org/10.1145/2485760.2485790

[28] Lee Jones, Meghrik Isagholi, Elizabeth Meiklejohn, Snow Xu, Kara Truskolawski, Jessica Hayon, Grace Jun, Pinar Guvenc, and Christina Mallon-Michalove. 2020 Hack-Ability: Using Co-Design to Develop an Accessible Toolkit for Adding Pockets to Garments. In Proceedings of the 16th Participatory Design Conference 2020-Participation (s) Otherwise-Volume 2. 95-99. https://doi.org/10.1145/3384772. 3385124

[29] Lee Jones, Sara Nabil, and Audrey Girouard. 2020. Swatch-bits: Prototyping E-textiles with Modular Swatches. In Proceedings of the Fourteenth International Conference on Tangible, Embedded, and Embodied Interaction. 893-897. https: //doi.org/10.1145/3374920.3374971

[30] Lee Jones, Sara Nabil, Amanda McLeod, and Audrey Girouard. 2020. Wearable Bits: scaffolding creativity with a prototyping toolkit for wearable e-textiles. In Proceedings of the Fourteenth International Conference on Tangible, Embedded, and Embodied Interaction. 165-177. https://doi.org/10.1145/3374920.3374954

[31] Majeed Kazemitabaar, Jason McPeak, Alexander Jiao, Liang He, Thomas Outing, and Jon E Froehlich. 2017. Makerwear: A tangible approach to interactive wearable creation for children. In Proceedings of the 2017 chi conference on human factors in computing systems. 133-145. https://doi.org/10.1145/3025453.3025887

[32] Majeed Kazemitabaar, Leyla Norooz, Mona Leigh Guha, and Jon E Froehlich. 2015. MakerShoe: towards a wearable e-textile construction kit to support creativity, playful making, and self-expression. In Proceedings of the 14th International Conference on Interaction Design and Children. 449-452. https://doi.org/10.1145/ 2771839.2771883

[33] Arounna Khounnoraj. 2019. Punch Needle. Quadrille.

[34] Konstantin Klamka, Raimund Dachselt, and Jürgen Steimle. 2020. Rapid IronOn User Interfaces: Hands-on Fabrication of Interactive Textile Prototypes. In Proceedings of the 2020 CHI Conference on Human Factors in Computing Systems. 1-14. https://doi.org/10.1145/3313831.3376220

[35] Nantia Koulidou, Jayne Wallace, Miriam Sturdee, and Abigail Durrant. 2020. Drawing on Experiences of Self: Dialogical Sketching. In Proceedings of the 2020 ACM Designing Interactive Systems Conference. 255-267. https://doi.org/10.1145/ 3357236.3395513

[36] Joanne Leong, Florian Perteneder, Hans-Christian Jetter, and Michael Haller. 2017. What a Life! Building a Framework for Constructive Assemblies. In Proceedings of the Eleventh International Conference on Tangible, Embedded, and Embodied Interaction. 57-66. https://doi.org/10.1145/3024969.3024985

[37] Jennifer C Mankoff, Eli Blevis, Alan Borning, Batya Friedman, Susan R Fussell, Jay Hasbrouck, Allison Woodruff, and Phoebe Sengers. 2007. Environmental sustainability and interaction. In CHI'07 extended abstracts on Human factors in computing systems. 2121-2124. https://doi.org/10.1145/1240866.1240963

[38] Fabrice Matulic, Brian Vogel, Naoki Kimura, and Daniel Vogel. 2019. Eliciting PenHolding Postures for General Input with Suitability for EMG Armband Detection. In Proceedings of the 2019 ACM International Conference on Interactive Surfaces and Spaces. 89-100. https://doi.org/10.1145/3343055.3359720

[39] David A Mellis, Sam Jacoby, Leah Buechley, Hannah Perner-Wilson, and Jie Qi. 2013. Microcontrollers as material: crafting circuits with paper, conductive ink, electronic components, and an" untoolkit". In Proceedings of the 7th International Conference on Tangible, Embedded and Embodied Interaction. 83-90. https://doi. org $/ 10.1145 / 2460625.2460638$

[40] Sara Nabil, Lee Jones, and Audrey Girouard. 2021. Soft Speakers: Digital Embroidering of DIY Customizable Fabric Actuators. In Fifteenth International Conference on Tangible, Embedded, and Embodied Interaction (TEI '21), February 14-17, 2021, Salzburg, Austria. Association for Computing Machinery, New York, NY, USA.

[41] Sara Nabil, Jan Kučera, Nikoletta Karastathi, David S Kirk, and Peter Wright. 2019. Seamless Seams: Crafting Techniques for Embedding Fabrics with Interactive Actuation. In Proceedings of the 2019 on Designing Interactive Systems Conference. 987-999. https://doi.org/10.1145/3322276.3322369

[42] Koya Narumi, Xinyang Shi, Steve Hodges, Yoshihiro Kawahara, Shinya Shimizu, and Tohru Asami. 2015. Circuit eraser: A tool for iterative design with conductive ink. In Proceedings of the 33rd Annual ACM Conference Extended Abstracts on Human Factors in Computing Systems. 2307-2312. https://doi.org/10.1145/ 2702613.2732876

[43] Grace Ngai, Stephen CF Chan, Hong Va Leong, and Vincent TY Ng. 2013. Designing $\mathrm{i}^{*}$ CATch: A multipurpose, education-friendly construction kit for physical and wearable computing. ACM Transactions on Computing Education (TOCE) 13, 2 (2013), 1-30. https://doi.org/10.1145/2483710.2483712

[44] Margaret Orth. 2006. Electronic textile touch light controller. US Patent 7,054,133.

[45] Rose Pearlman. 2019. Modern Rug Hooking. Roost Books.

[46] Andreas Peetz, Konstantin Klamka, and Raimund Dachselt. 2019. BodyHub: A Reconfigurable Wearable System for Clothing. In The Adjunct Publication of the 32nd Annual ACM Symposium on User Interface Software and Technology. 39-41. https://doi.org/10.1145/3332167.3357108

[47] Hannah Perner-Wilson, Leah Buechley, and Mika Satomi. 2010. Handcrafting textile interfaces from a kit-of-no-parts. In Proceedings of the fifth international conference on Tangible, embedded, and embodied interaction. 61-68. https://doi. org/10.1145/1935701.1935715

[48] Irene Posch. 2017. Crafting Tools for Textile Electronic Making. In Proceedings of the 2017 CHI Conference Extended Abstracts on Human Factors in Computing Systems. 409-412. https://doi.org/10.1145/3027063.3052972

[49] Irene Posch and Geraldine Fitzpatrick. 2018. Integrating Textile Materials with Electronic Making: Creating New Tools and Practices. In Proceedings of the Twelfth International Conference on Tangible, Embedded, and Embodied Interaction. 158-165. https://doi.org/10.1145/3173225.3173255

[50] Irene Posch, Liza Stark, and Geraldine Fitzpatrick. 2019. eTextiles: reviewing a practice through its tool/kits. In Proceedings of the 23rd International Symposium on Wearable Computers. 195-205. https://doi.org/10.1145/3341163.3347738 
[51] Leanne Prain. 2014. Strange material: Storytelling through textiles. Arsenal Pulp Press.

[52] Jie Qi and Leah Buechley. 2014. Sketching in circuits: designing and building electronics on paper. In Proceedings of the SIGCHI Conference on Human Factors in Computing Systems. 1713-1722. https://doi.org/10.1145/2556288.2557391

[53] Jie Qi, Andrew "bunnie" Huang, and Joseph Paradiso. 2015. Crafting technology with circuit stickers. In Proceedings of the 14th International Conference on Interaction Design and Children. 438-441. https://doi.org/10.1145/2771839.2771873

[54] Barath Raghavan and Daniel Pargman. 2017. Means and ends in human-computer interaction: Sustainability through disintermediation. In Proceedings of the 2017 CHI Conference on Human Factors in Computing Systems. 786-796. https://doi. org $/ 10.1145 / 3025453.3025542$

[55] Analisa Russo, Bok Yeop Ahn, Jacob J Adams, Eric B Duoss, Jennifer T Bernhard, and Jennifer A Lewis. 2011. Pen-on-paper flexible electronics. Advanced materials 23, 30 (2011), 3426-3430.

[56] Sarah Schoemann and Michael Nitsche. 2017. Needle as input: exploring practice and materiality when crafting becomes computing. In Proceedings of the Eleventh International Conference on Tangible, Embedded, and Embodied Interaction. 299308. https://doi.org/10.1145/3024969.3024999

[57] Kimberly Sheridan, Erica Rosenfeld Halverson, Breanne Litts, Lisa Brahms, Lynette Jacobs-Priebe, and Trevor Owens. 2014. Learning in the making: A comparative case study of three makerspaces. Harvard Educational Review 84, 4 (2014), 505-531.

[58] Angelika Strohmayer and Janis Meissner. 2017. We had tough times, but we've sort of sewn our way through it: the partnership quilt. XRDS: Crossroads, The ACM Magazine for Students 24, 2 (2017), 48-51. https://doi.org/10.1145/3155128
[59] Paul Strohmeier, Narjes Pourjafarian, Marion Koelle, Cedric Honnet, Bruno Fruchard, and Jürgen Steimle. 2020. Sketching On-Body Interactions using PiezoResistive Kinesiology Tape. In Proceedings of the Augmented Humans International Conference. $1-7$. https://doi.org/10.1145/3384657.3384774

[60] Miriam Sturdee, Aluna Everitt, Joseph Lindley, Paul Coulton, and Jason Alexander. 2019. Visual Methods for the Design of Shape-Changing Interfaces. In IFIP Conference on Human-Computer Interaction. Springer, 337-358. https://doi.org/ 10.1007/s00779-010-0311-y

[61] Miriam Sturdee, John Hardy, Nick Dunn, and Jason Alexander. 2015. A public ideation of shape-changing applications. In Proceedings of the 2015 International Conference on Interactive Tabletops \& Surfaces. 219-228. https://doi.org/10.1145/ 2817721.2817734

[62] Miriam Sturdee, Makayla Lewis, and Nicolai Marquardt. 2018. Feeling SketCHI? the lasting appeal of the drawn image in HCI. interactions 25, 6 (2018), 64-69. https://doi.org/10.1145/3274562

[63] Miriam Sturdee and Joseph Lindley. 2019. Sketching \& Drawing as Future Inquiry in HCI. In Proceedings of the Halfway to the Future Symposium 2019.1-10. https: //doi.org/10.1145/3363384.3363402

[64] Patrick A Tresset and F Fol Leymarie. 2012. Sketches by Paul the robot. In Proceedings of the eighth annual symposium on computational aesthetics in graphics, visualization, and imaging. 17-24.

[65] Jagoda Walny, Samuel Huron, and Sheelagh Carpendale. 2015. An exploratory study of data sketching for visual representation. In Computer Graphics Forum, Vol. 34. Wiley Online Library, 231-240. https://doi.org/10.1111/cgf.12635

[66] Shanel Wu and Laura Devendorf. 2020. Unfabricate: Designing Smart Textiles for Disassembly. In Proceedings of the 2020 CHI Conference on Human Factors in Computing Systems. 1-14. https://doi.org/10.1145/3313831.3376227 